\title{
Inflammation and $\mathrm{N}$-formyl peptide receptors mediate the angiogenic activity of human vitreous humour in proliferative diabetic retinopathy
}

\author{
Sara Rezzola ${ }^{1}$ - Michela Corsini ${ }^{1} \cdot$ Paola Chiodelli $^{1}$ - Anna Cancarini ${ }^{2}$. \\ Imtiaz M. Nawaz ${ }^{1}$ - Daniela Coltrini ${ }^{1} \cdot$ Stefania Mitola $^{1} \cdot$ Roberto Ronca $^{1}$. \\ Mirella Belleri $^{1} \cdot$ Liliana Lista $^{3}$ - Dario Rusciano ${ }^{4}$ - Mario De Rosa ${ }^{5} \cdot$ Vincenzo Pavone $^{3}$. \\ Francesco Semeraro $^{2} \cdot$ Marco Presta $^{1}$
}

Received: 3 September 2016/Accepted: 13 December 2016/Published online: 13 January 2017

(C) Springer-Verlag Berlin Heidelberg 2017

\begin{abstract}
Aims/hypothesis Angiogenesis and inflammation characterise proliferative diabetic retinopathy (PDR), a major complication of diabetes mellitus. However, the impact of inflammation on the pathogenesis of PDR neovascularisation has not been elucidated. Here, we assessed the capacity of PDR vitreous fluid to induce pro-angiogenic/proinflammatory responses in endothelium and the contribution of the inflammation-related pattern recognition $N$-formyl peptide receptors (FPRs) in mediating these responses.

Methods Pooled and individual pars plana vitrectomy-derived PDR vitreous fluid ('PDR vitreous') samples were assessed in endothelial cell proliferation, motility, sprouting and
\end{abstract}

Electronic supplementary material The online version of this article (doi:10.1007/s00125-016-4204-0) contains peer-reviewed but unedited supplementary material, which is available to authorised users.

Sara Rezzola

sara.rezzola@unibs.it

Francesco Semeraro

francesco.semeraro@unibs.it

Marco Presta

marco.presta@unibs.it

1 Department of Molecular and Translational Medicine, University of Brescia, Via Branze 39, Brescia 25123, Italy

2 Department of Ophthalmology, University of Brescia, Piazzale Spedali Civili 1, Brescia 25123, Italy

3 Department of Chemical Sciences, 'Federico II' University of Naples, Naples, Italy

4 Sooft Italia Spa, Montegiorgio, Italy

5 Department of Experimental Medicine, Second University of Naples, Naples, Italy morphogenesis assays, and for the capacity to induce proinflammatory transcription factor activation, reactive oxygen species production, intercellular junction disruption and leucocyte-adhesion molecule upregulation in these cells. In vivo, the pro-angiogenic/proinflammatory activity of PDR vitreous was tested in murine Matrigel plug and chick embryo chorioallantoic membrane (CAM) assays. Finally, the FPR inhibitors Boc-Phe-Leu-Phe-Leu-Phe (Boc-FLFLF) and Ac-L-Arg-Aib-L-Arg-L-C $\alpha(\mathrm{Me}) \mathrm{Phe}-\mathrm{NH}_{2}$ tetrapeptide (UPARANT) were evaluated for their capacity to affect the biological responses elicited by PDR vitreous.

Results PDR vitreous activates a pro-angiogenic/proinflammatory phenotype in endothelial cells. Accordingly, PDR vitreous triggers a potent angiogenic/inflammatory response in vivo. Notably, the different capacity of individual PDR vitreous samples to induce neovessel formation in the CAM correlates with their ability to recruit infiltrating $\mathrm{CD} 45^{+}$cells. Finally, the FPR inhibitor Boc-FLFLF and the novel FPR antagonist UPARANT inhibit neovessel formation and inflammatory responses triggered by PDR vitreous in the CAM assay.

Conclusions/interpretation This study provides evidence that inflammation mediates the angiogenic activity of PDR vitreous and paves the way for the development of FPR-targeting antiinflammatory/anti-angiogenic approaches for PDR therapy.

Keywords Angiogenesis $\cdot$ Diabetic retinopathy $\cdot$ Endothelial cells $\cdot$ Inflammation $\cdot N$-formyl peptide receptor $\cdot$ Vitreous
Abbreviations
Boc-FLFLF Boc-Phe-Leu-Phe-Leu-Phe
CAM Chorioallantoic membrane
fMLF Formyl-methionyl-leucyl phenylalanine 


\begin{tabular}{|c|c|}
\hline FPR & $N$-formyl peptide receptor \\
\hline ICAM-1 & Intercellular adhesion molecule 1 \\
\hline pCREB & $\begin{array}{l}\text { Phosphorylated cAMP-response element } \\
\text { binding protein }\end{array}$ \\
\hline PDR & Proliferative diabetic retinopathy \\
\hline $\mathrm{CR}$ & Quantitative PCR \\
\hline OS & Reactive oxygen species \\
\hline & Urokinase-type plasminogen activator \\
\hline AR & $\begin{array}{l}\text { Urokinase-type plasminogen activator } \\
\text { receptor }\end{array}$ \\
\hline ARANT & $\begin{array}{l}\text { Ac-L-Arg-Aib-L-Arg-L-C } \alpha(\mathrm{Me}) \mathrm{Phe}-\mathrm{NH}_{2} \\
\text { tetrapeptide }\end{array}$ \\
\hline AM-1 & Vascular cell adhesion molecule- 1 \\
\hline$-\mathrm{CAD}$ & Vascular endothelial cadherin \\
\hline & Vascular endothelial growth factor \\
\hline & Tight junction protein 1 \\
\hline
\end{tabular}

\section{Introduction}

Diabetic retinopathy, the leading cause of visual impairment in the working-age population [1], begins as non-proliferative retinal abnormalities and progresses to proliferative diabetic retinopathy (PDR) characterised by neovascularisation and a persistent grade of inflammation [2-5]. Even though antiangiogenic vascular endothelial growth factor (VEGF) inhibitors are widely used in PDR therapy [2], several limitations to anti-VEGF interventions exist [6]. Furthermore, production of other angiogenic factors and proinflammatory mediators may nullify and/or cause resistance to anti-VEGF therapies $[6,7]$.

Angiogenesis and inflammation are closely related processes that play a pivotal role in ocular diseases associated with retinal neovascularisation $[5,8,9]$. Thus, a tight cross talk may exist between angiogenesis and inflammation in PDR. Accordingly, the therapeutic potential of intravitreal administration of anti-inflammatory corticosteroids (e.g. triamcinolone acetonide) has been investigated in patients with diabetic retinopathy. However, beneficial effects can be transient and associated with steroid-related adverse events [10, 11]. This calls for a better understanding of the cross talk between angiogenesis and inflammation in PDR to identify novel anti-inflammatory approaches able to suppress retinal neovascularisation.

$N$-formyl peptide receptors (FPRs) are involved in the recruitment and activation of immune cells in response to pathogen-associated molecular patterns. In addition, experimental evidence implicates FPRs in angiogenic responses linked to inflammation [12], and FPR interaction with the urokinase-type plasminogen activator (uPA)/uPA receptor (uPAR) system may stimulate angiogenesis in a proteaseindependent manner [13-15]. Accordingly, the uPARderived tetrapeptide Ac-L-Arg-Aib-L-Arg-L-C $\alpha(\mathrm{Me}) \mathrm{Phe}-$ $\mathrm{NH}_{2}$ (UPARANT) competes with formyl-methionyl-leucyl phenylalanine (fMLF) peptide for binding to FPRs and is endowed with a significant anti-angiogenic activity in vitro and in vivo $[16,17]$. In addition, UPARANT prevents ocular angiogenesis and reduces the levels of inflammatory mediators in murine models of oxygen-induced retinopathy and laser-induced choroidal neovascularisation $[17,18]$.

The study of the biological effects exerted by PDR vitreous fluid ('PDR vitreous') on endothelial cells may represent a useful tool to investigate the relationship between neovascular and inflammatory responses in preclinical experimental models. PDR vitreous contains high levels of pro-angiogenic and proinflammatory mediators $[19,20]$ and stimulates angiogenesis in vitro and in vivo, whereas no angiogenic response is elicited by vitreous fluid from patients with a macular hole [7, 17, 21-23]. However, no data are available about the capacity of PDR vitreous to exert proinflammatory responses in endothelium, their relationship with its ability to stimulate neovessel formation or the role of FPRs in this process. This study was designed to assess the pro-angiogenic and proinflammatory potential of vitreous fluid obtained from PDR patients after pars plana vitrectomy and to evaluate the impact of FPR inhibitors on their biological activity.

\section{Methods}

Reagents A list of reagents is included in the electronic supplementary material (ESM).

Human vitreous fluid samples Patients with PDR (Table 1) and age-matched patients with macular hole underwent pars plana vitrectomy at the Clinics of Ophthalmology (University of Brescia) during the period January 2014-June 2016. Collection and analysis of human samples were approved by the internal review board of the Spedali Civili of Brescia and followed the principles of the Declaration of Helsinki. Informed consent was obtained from all participants. Samples were stored at $-80^{\circ} \mathrm{C}$. Their content of pro-angiogenic/proinflammatory mediators was evaluated using a semiquantitative antibody-based RayBio Human Angiogenesis Array C1000 (Raybiotech, Norcross, GA, USA). See ESM methods.

All assays were performed on vitreous samples pooled from 4-5 patients unless specified otherwise. Data are representative of at least three independent pools of vitreous fluid that provided similar results.

In vitro and ex vivo endothelial cell studies PDR vitreous was tested on HUVECs in different angiogenesis assays. Briefly, in the proliferation assay, HUVECs $(17,000$ cells/ $\mathrm{cm}^{2}$ ) were treated with increasing amounts of vitreous fluid diluted in culture medium plus $2.5 \% \mathrm{FCS}$ and counted after $24 \mathrm{~h}$. In the migration assay, HUVECs were seeded at 
Table 1 Characteristics of patients with PDR

\begin{tabular}{|c|c|c|}
\hline Characteristic & $\begin{array}{l}\text { Patients for pooled } \\
\text { vitreous humour assays }\end{array}$ & $\begin{array}{l}\text { Patients for individual } \\
\text { vitreous humour assays }\end{array}$ \\
\hline Patients/eyes & $29 / 29$ & $24 / 25$ \\
\hline \multicolumn{3}{|l|}{ Clinical features } \\
\hline Sex (male) & 17 & 16 \\
\hline Age $(\text { years })^{\text {a }}$ & $66 \pm 16$ & $64 \pm 16$ \\
\hline Type $1 /$ type 2 diabetes & $5 / 24$ & $5 / 20$ \\
\hline Insulin treatment & 24 & 19 \\
\hline Nephropathy & 7 & 7 \\
\hline Hypertension & 22 & 20 \\
\hline Dyslipidaemia & 10 & 11 \\
\hline \multicolumn{3}{|l|}{ Ophthalmological features } \\
\hline PDR & 29 & 25 \\
\hline PDR with vitreous haemorrhage & 16 & 17 \\
\hline PDR with macular oedema & 5 & 5 \\
\hline \multicolumn{3}{|l|}{ Therapies } \\
\hline Intravitreal injection of anti-VEGF blocker & 10 & 13 \\
\hline Pan-retinal laser photocoagulation & 17 & 20 \\
\hline
\end{tabular}

Data are $n$ unless indicated otherwise

${ }^{\mathrm{a}}$ Mean $\pm \mathrm{SD}$
$1.0 \times 10^{6} \mathrm{cells} / \mathrm{ml}$ in the upper compartment of a Boyden chamber, and increasing amounts of vitreous fluid were placed in the lower compartment. After $3 \mathrm{~h}$ at $37^{\circ} \mathrm{C}$, cells that had migrated to the lower side of the filter were counted. In the morphogenesis assay, HUVECs were seeded on Matrigel at $2.0 \times 10^{5}$ cells $/ \mathrm{ml}$ in the absence or presence of vitreous fluid and the number of meshes was counted after $8 \mathrm{~h}$. In the endothelial cell sprouting assay, fibrin gel-embedded HUVEC spheroids were stimulated with vitreous fluid and endothelial cell sprouts were counted $24 \mathrm{~h}$ thereafter. In addition, murine retina tissue fragments were embedded in fibrin gel and stimulated with vitreous fluid. Retinal endothelial cell sprouting was evaluated after 7 days. See ESM methods for details.

The same vitreous samples were assessed in HUVECs for their proinflammatory potential. Briefly, HUVECs $\left(1.0 \times 10^{6}\right)$ suspended in serum-free medium in the presence of 1:4 vitreous fluid were incubated with $5 \mu \mathrm{mol} / 1$ dichloro-dihydrofluorescein diacetate (DCFH-DA) for 5-30 $\mathrm{min}$ in the dark at $37^{\circ} \mathrm{C}$ and reactive oxygen species (ROS) production was quantified by FACS analysis. Serum-starved confluent HUVECs were treated with 1:4 vitreous fluid for $0-30 \mathrm{~min}$, fixed in $4 \%$ paraformaldehyde and immunostained with antibodies against anti-tight junction protein 1 (ZO-1), antivascular endothelial cadherin (VE-CAD), antiphosphorylated-cAMP-response element-binding protein (pCREB) or anti-NF-kB p65 subunit. Endothelial barrier function was studied on confluent HUVEC monolayers by a trans-endothelial electrical resistance assay in which impedance was monitored every min for $3 \mathrm{~h}$ following PDR vitreous treatment. Also, fluorescently labelled THP-1 cells $\left(7.0 \times 10^{5}\right.$ cells/well $)$ were added on the top of HUVEC monolayers that had been treated with 1:4 vitreous fluid for $18 \mathrm{~h}$. After $30 \mathrm{~min}$ at $37^{\circ} \mathrm{C}$, adherent THP-1 cells were quantified. See ESM methods for details.

Finally, HUVECs were evaluated for FPR1-3 expression by RT-PCR, FACS and western blot analysis according to standard procedures. See ESM methods.

Real-time quantitative PCR Real-time quantitative PCR (qPCR) was used to determine the relative expression levels of mRNAs. See ESM methods.

Animal experiments The pro-angiogenic/proinflammatory activity of vitreous fluid was evaluated in vivo in the murine Matrigel plug [24] and chick embryo chorioallantoic membrane (CAM) [7] assays.

Matrigel was mixed at $4^{\circ} \mathrm{C}$ with 1:4 vitreous fluid and injected subcutaneously $(0.4 \mathrm{ml} /$ mouse $)$ into the flank of 6-8 week old C57BL6 female mice (Charles River, Calco, Italy). One week after injection, the proinflammatory/proangiogenic response was quantified in harvested plugs by qPCR. See ESM methods.

Alginate beads $(3 \mu \mathrm{l})$ containing $2 \mu \mathrm{l}$ of vitreous fluid were placed on top of the CAM of fertilised chicken eggs at day 11 of development. Microvessels converging towards the implant were counted under a stereomicroscope and the $\mathrm{CD} 5^{+}$infiltrate was assessed by immunofluorescence analysis. See ESM methods. 
Procedures were carried out according to the Guide for the care and use of laboratory animals, the animal care Italian guidelines (DL 116/92) and the European Communities Council Directive (86/609/EEC) and were approved by the Ethical Committee in Animal Experiments of the University of Brescia.

Statistics Data are mean \pm SEM. Statistical significance was evaluated with commercial software (GraphPad Prism 6; San Diego, CA, USA) using Student's $t$ test or one-way ANOVA followed by Bonferroni multiple comparison post test. Differences were considered significant when $p<0.05$.

\section{Results}

Human PDR vitreous induces a pro-angiogenic/proinflammatory phenotype in endothelial cells Three pools of human PDR vitreous were evaluated for their content of proangiogenic/proinflammatory mediators using a semiquantitative antibody-based array. As shown in ESM Table 2, a variety of cytokines, chemokines and angiogenic growth factors are detectable in PDR vitreous, supporting the hypothesis that this fluid may exert both angiogenic and inflammatory responses in endothelial cells.

On this basis, PDR vitreous was assessed for its capacity to affect in vitro different steps of the angiogenic process. As shown in Fig. 1, PDR vitreous induces HUVEC proliferation and motility, endothelial cell sprouting in a three-dimensional murine retinal angiogenesis assay, and capillary-like tube morphogenesis in HUVECs seeded on Matrigel. No activity was exerted by the vitreous from age-matched patients with macular hole, thus confirming the specificity of the effect.

Next, we analysed the capacity of PDR vitreous to activate a proinflammatory phenotype in endothelial cells. As shown

Fig. 1 PDR vitreous induces a pro-angiogenic phenotype in endothelial cells. Pooled vitreous samples from patients with PDR (white symbols) and macular hole (black symbols) were tested using angiogenesis-related endothelial cell assays ( $n \geq 3$ ): (a) HUVEC proliferation; (b) HUVEC migration; (c) murine retina fragment sprouting (untreated, grey symbols); and (d) HUVEC morphogenesis on Matrigel. (e, $\mathbf{f}$ Control (e) and PDR vitreoustreated (f) HUVECs seeded on Matrigel. Data are means \pm SEM. ${ }^{*} p<0.05 ; * *<<0.01$ vs control. Scale bars, $1 \mathrm{~mm}$. MH, macular hole in Fig. 2a-d, PDR vitreous induces the rapid nuclear translocation of the proinflammatory transcription factors $N F-\kappa B$ and pCREB in HUVECs, whereas no pCREB translocation was observed in cells treated with vitreous from patients with macular hole (ESM Fig. 1). Moreover, PDR vitreous stimulates the early production of ROS in HUVECs (Fig. 2e).

The loss of endothelial barrier integrity and leucocyte adhesion are early events induced by proinflammatory mediators in endothelial cells. HUVEC monolayers treated with PDR vitreous show an increased number of intercellular gaps and the loss of ZO-1 and VE-CAD junction protein organisation (Fig. 2f). Accordingly, PDR vitreous affects endothelial barrier function as assessed by a trans-endothelial electrical resistance assay performed on HUVEC monolayers (Fig. $2 \mathrm{~g}$ ). In addition, PDR vitreous upregulates the expression of the leucocyteadhesion receptors E-SELECTIN, vascular cell adhesion molecule 1 (VCAM-1) and intercellular adhesion molecule 1 (ICAM-1) in HUVECs, with no effect on P-SELECTIN (Fig. 2h). Eventually, PDR vitreous increases the adhesion of human monocytic THP-1 cells to a HUVEC monolayer, this increase being inhibited by neutralising VCAM-1 plus ICAM-1 antibodies (Fig. 2i).

Pharmacological blockade of inflammation hampers the angiogenic activity exerted in vivo by human PDR vitreous The capacity of PDR vitreous to induce angiogenic and inflammatory responses in vivo was investigated in a murine Matrigel plug assay. PDR vitreous-treated plugs showed a significant upregulation of the expression of endothelial Cd31 (also known as Pecam1) and leucocyte Cd45 (also known as Ptprc) transcripts in respect to control plugs (Fig. 3a). Histological analysis confirmed an increase of $\mathrm{CD} 31^{+}$neovessels and of infiltrating $\mathrm{CD} 45^{+}$cells in PDR vitreous-treated vs control plugs (Fig. 3b). In addition, qPCR
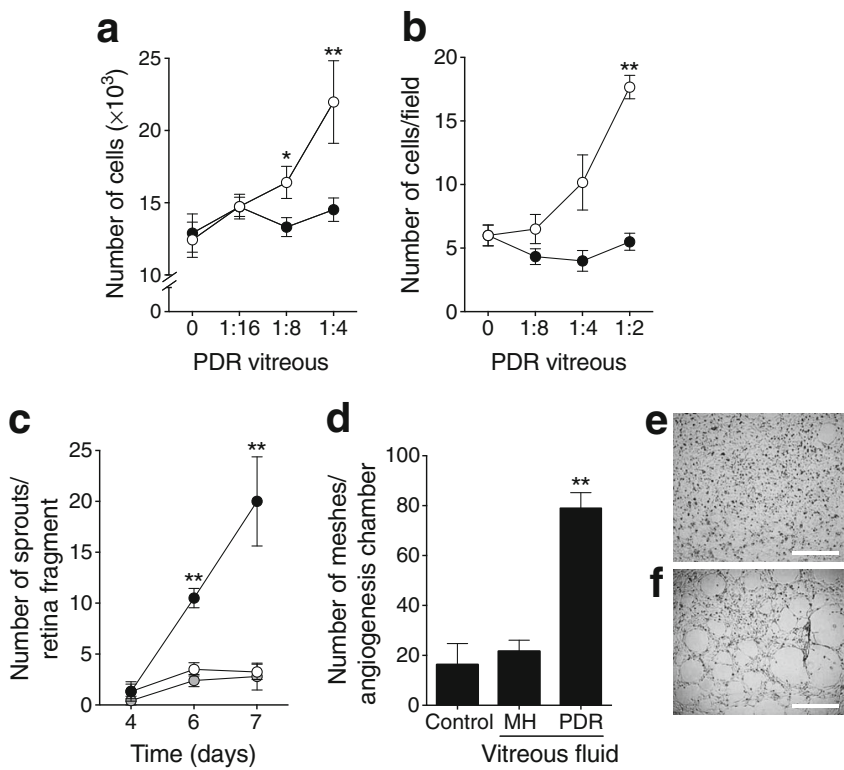

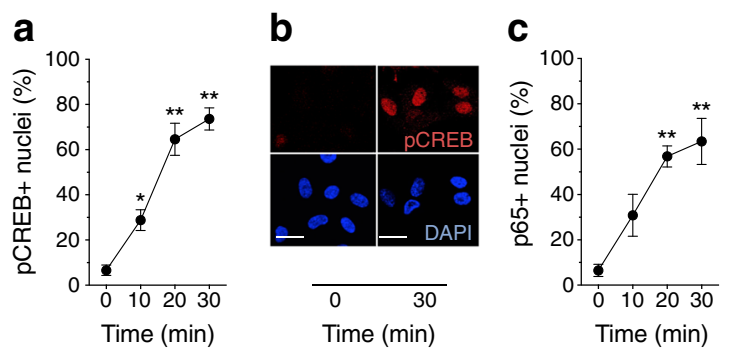

d

e

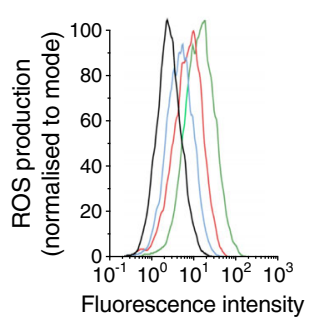

f

g

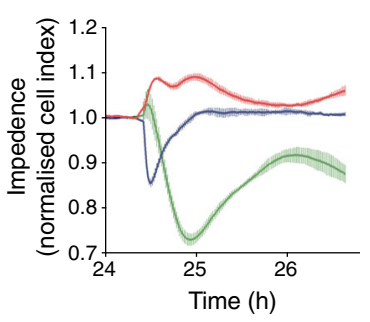

h
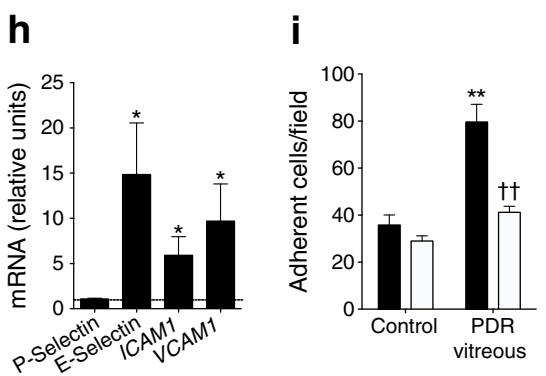

Fig. 2 PDR vitreous exerts a proinflammatory activity in endothelial cells. $(\mathbf{a}, \mathbf{c})$ The percentages of pCREB-immunoreactive (a) and NF-KB p65 subunit-immunoreactive (c) HUVEC nuclei ( $n=60$ cells). (b, d) pCREB (b) and NF- $\mathrm{kB}$ p65 subunit (d) immunoreactivity (red) in HUVECs at 0 (left panels) and 30 min (right panels) after PDR vitreous treatment; nuclei were stained with DAPI (blue). (e) ROS production by HUVECs. Data are representative of three determinations. (f) Junctional protein organisation in HUVECs. ZO-1 (red) and VE-CAD (green) immunoreactivity; nuclei were stained with DAPI (blue). Data are

representative of three experiments. (g) Trans-endothelial electrical resistance in untreated (red), $2 \mathrm{mg} / \mathrm{ml} \mathrm{BSA-treated} \mathrm{(blue)} \mathrm{or} \mathrm{PDR} \mathrm{vitreous-}$ treated (green) HUVEC monolayers. (h) qPCR analysis. Fold increase in the expression levels of target genes in PDR vitreous-treated HUVECs $(n=4)$. (i) THP- 1 cell adhesion on HUVEC monolayers $(n=3)$. Black bars, untreated; white bars, anti-VCAM + anti-ICAM antibodies. Data are means \pm SEM. ${ }^{*} p<0.05 ; * * p<0.01$ vs control; ${ }^{\dagger} p<0.01$ vs untreated. Scale bars, $25 \mu \mathrm{m}$

analysis of the expression levels of various cell lineagespecific markers indicated that the inflammatory infiltrate in PDR vitreous plugs was characterised by an increased recruitment of chondroitin sulphate proteoglycan $4(\mathrm{NG} 2)^{+}$pericytes and $\mathrm{CD} 11 \mathrm{~B}^{+}$monocytes, with an abundance of the expression of the M2 macrophage markers $E g r 2$ and $C d 83$ [25]. At variance, no changes in the expression levels of the M1 macrophage marker $C d 38$ [25] was observed between control and PDR vitreous plugs (Fig. 3a). Lymphocyte antigen 6 complex, locus $\mathrm{G}(\mathrm{GR} 1)^{+}$neutrophils, $\mathrm{CD} 4^{+}$and $\mathrm{CD} 8^{+} \mathrm{T}$ lymphocytes, $\mathrm{CD} 19^{+} \mathrm{B}$ lymphocytes and $\mathrm{CD} 161^{+}$natural killer cells/ lymphocytes were scarcely present in both experimental groups, as indicated by the negligible levels of expression of the corresponding markers that were detected by qPCR analysis only after an unreliable, very high number of amplification cycles (data not shown).

To further assess the proangiogenic/proinflammatory activity of PDR vitreous, alginate beads containing $2.0 \mu \mathrm{l} /$ pellet of
PDR vitreous were implanted onto the top of the chick embryo CAM. Numerous neovessels converging towards the implant were detectable $72 \mathrm{~h}$ after grafting when compared with vehicle-treated embryos (Fig. 3c, d). Furthermore, PDR vitreous-loaded beads recruited a remarkable mononuclear $\mathrm{CD} 45^{+}$cell infiltrate in the surrounding mesenchyme, representing embryonic proinflammatory monocytes/ macrophages [26], barely detectable in control implants (Fig. 3c, d). Notably, analysis of the kinetics of response of the chick embryo CAM highlights the sharp increase in $\mathrm{CD} 45^{+}$cell recruitment following PDR vitreous plug implantation with a slower appearance of the newly formed blood vessels (Fig. 3e). In addition, a significant positive correlation ( $p=0.01$ ) was observed between the number of infiltrating $\mathrm{CD} 45^{+}$cells and the number of neovessels when samples of vitreous fluid obtained from ten PDR patients were individually tested in the CAM assay, more angiogenic samples being characterised by a stronger inflammatory response (Fig. 3f). 


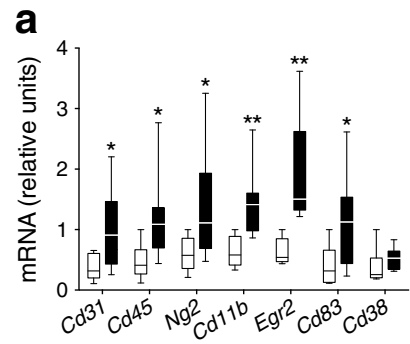

b

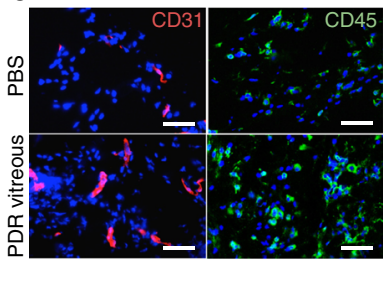

C

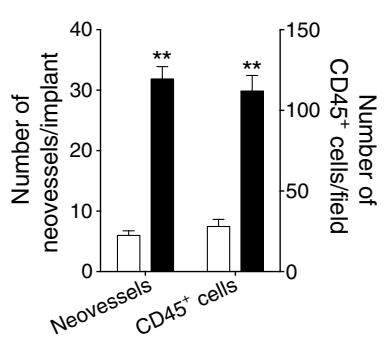

d

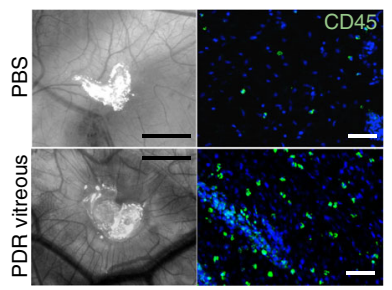

e

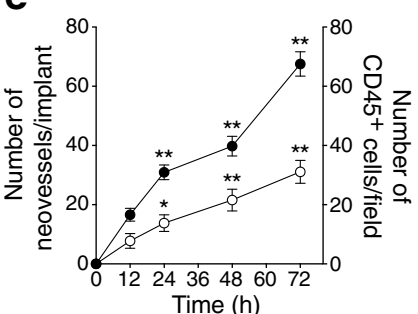

f

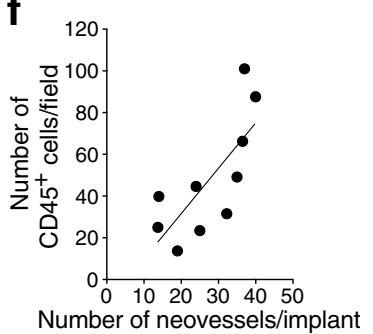

a

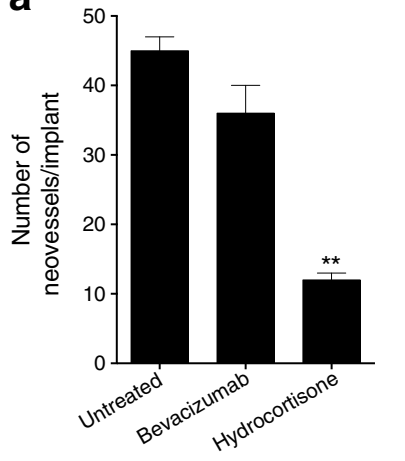

b
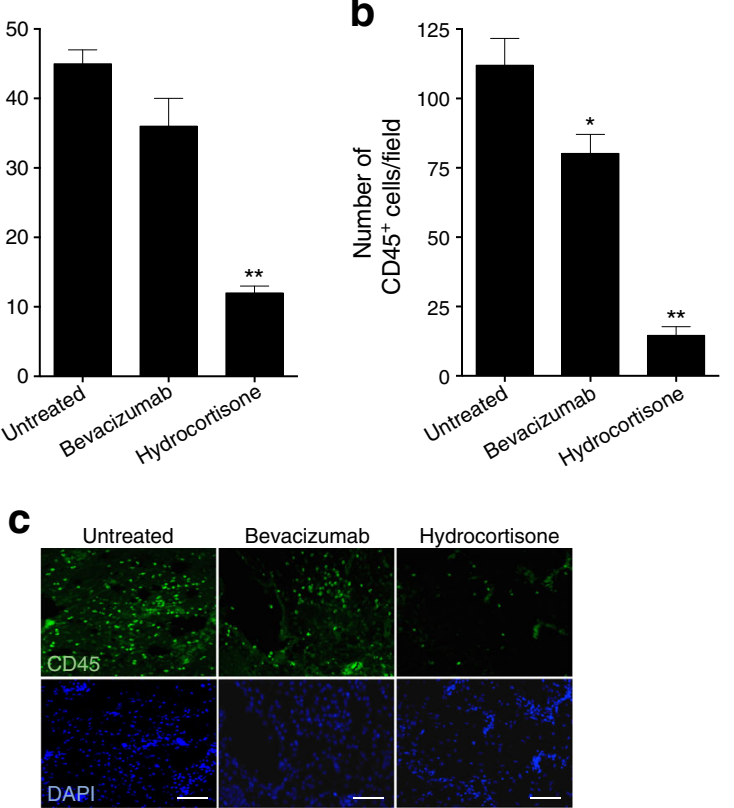

Fig. 4 Hydrocortisone inhibits PDR vitreous-induced neovascularisation. Chick embryo CAMs were implanted with PDR vitreous-alginate beads in the absence or presence of bevacizumab or hydrocortisone. The number of neovessels/implant $(n=8)(\mathbf{a})$ and $\mathrm{CD} 45^{+}$cells/microscopic field $(n=25)$ infiltrating the CAM mesenchyme (b). (c) CD45 ${ }^{+}$infiltrate (green) in CAMs; nuclear staining with DAPI (blue). Data are means \pm SEM. $* p<0.05 ; * *<<0.01$ vs untreated. Scale bars, $50 \mu \mathrm{m}$

Fig. 3 PDR vitreous induces an angio-inflammatory response in vivo. (a, b) Murine Matrigel plug assay. (a) qPCR analysis of the expression levels of cell lineage-specific markers in the plugs. White bars, PBS; black bars, PDR vitreous ( $n=12)$. (b) CD31 (red) and CD45 infiltrate (green) in PBS and PDR vitreous Matrigel plugs; nuclei were stained with DAPI (blue). (c-e) Chick embryo CAM assay. (c) Number of neovessels/implant $(n=8)$ and of $\mathrm{CD} 45^{+}$cells/microscopic field $(n=25)$ infiltrating the CAM mesenchyme. White bars, PBS; black bars, PDR vitreous. (d) Neovessels (left panels) and $\mathrm{CD}^{+} 5^{+}$infiltrate (green) (right panels) in PBS and PDR vitreous-treated CAMs; nuclei were stained with DAPI (blue). (e) Kinetics of neovessel formation (white symbols, $n=8$ ) and $\mathrm{CD} 45^{+}$cell recruitment (black symbols, $n=25$ ) in PDR vitreous-stimulated CAMs. (f) Positive correlation between the number of neovessels and of infiltrating CD $45^{+}$ cells in CAMs treated with ten individual PDR vitreous samples; $p=0.01$, $r^{2}=0.6$. Data are means \pm SEM. $* p<0.05 ; * * p<0.01$ vs PBS. Scale bars, $50 \mu \mathrm{m}$ (white) and $2 \mathrm{~mm}$ (black)

To assess the role exerted by inflammation in the angiogenic response elicited in vivo by PDR vitreous, a series of CAM assays were performed in the absence and presence of the antiangiogenic prototypic VEGF blocker bevacizumab or the prototypic steroidal anti-inflammatory drug hydrocortisone. As shown in Fig. 4, hydrocortisone $(1.0 \mu \mathrm{g} /$ pellet $)$ caused a potent inhibition of the angiogenic response triggered by PDR vitreous that was paralleled by a significant reduction in the inflammatory infiltrate, whereas bevacizumab $(50 \mu \mathrm{g} /$ pellet $)$ exerted only a marginal effect. Together, these observations point to a non-redundant role of inflammation in the angiogenic process triggered by PDR vitreous.
UPARANT inhibits the angiogenic/inflammatory activity exerted by human PDR vitreous FPRs are implicated in the regulation of innate immune responses, inflammation, tissue repair and angiogenesis. Semi-quantitative RT-PCR, FACS and western blot analyses indicate that our HUVEC primary pared with monocytic THP-1 cells that express all three FPRs (ESM Fig. 2). In order to assess a possible involvement of FPRs in PDR, we evaluated the effect of the pan-FPR antagonist Boc-Phe-Leu-Phe-Leu-Phe (Boc-FLFLF) on the angiogenic/inflammatory potential of PDR vitreous. As shown in Fig. 5a, b, Boc-FLFLF inhibits PDR vitreousstimulated invasion of three-dimensional fibrin gel by HUVEC spheroids, whereas the selective FPR1 antagonist ciclosporin $\mathrm{H}$ and the FPR2 antagonist WRW4 were ineffective. In addition, Boc-FLFLF prevents neovascularisation and recruitment of $\mathrm{CD} 45^{+}$inflammatory cells triggered by PDR vitreous in the CAM assay (Fig. 5c, d). Together, these data point to FPRs as a potential therapeutic target in PDR.

The uPAR-derived tetrapeptide UPARANT is a novel FPR antagonist endowed with a significant anti-angiogenic activity. On this basis, we assessed the capacity of UPARANT to affect the angiogenic response induced in the chick embryo CAM by individual vitreous fluid samples obtained from 20 PDR patients. As shown in Fig. 6a, b, UPARANT $(1.0 \mu \mathrm{g} /$ pellet) significantly inhibited neovessel formation induced by cultures express FPR3 but not FPR1 and FPR2 when com- 


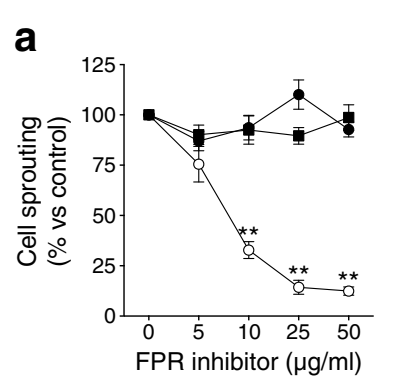

b

C

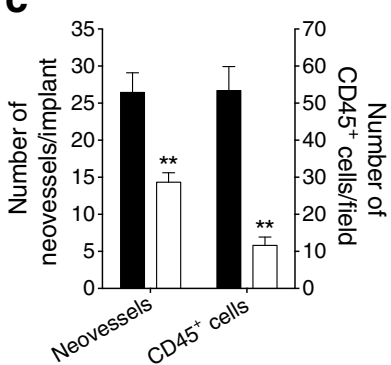

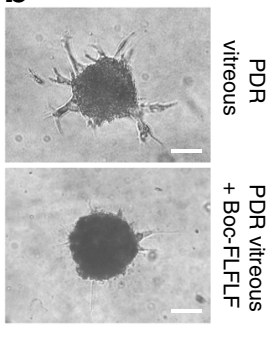

d

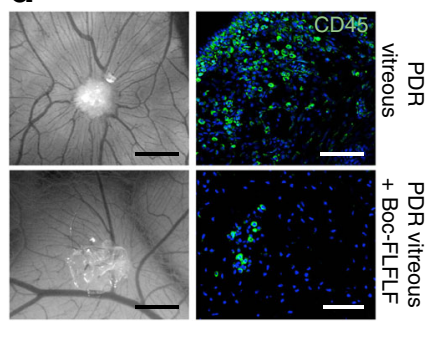

Fig. 5 Boc-FLFLF suppresses the angio-inflammatory responses induced by PDR vitreous. (a, b) HUVEC sprouting assay. (a) Endothelial cell sprouts in PDR vitreous-treated spheroids incubated with Boc-FLFLF (white circles), ciclosporine H (black circles) or WRW4 (black squares) $(n=3)$. Vitreous alone was used as control. (b) PDR vitreous-treated spheroids in the absence or in the presence of Boc-FLFLF. (c, d) Chick embryo CAM assay. (c) Number of neovessels/implant $(n=8)$ and of $\mathrm{CD} 5^{+}$cells/microscopic field $(n=25)$ infiltrating the CAM mesenchyme. Black bars, PDR vitreous; white bars, PDR vitreous + BocFLFLF. (d) Neovessels (left panels) and CD45 ${ }^{+}$infiltrate (green) (right panels) in PDR vitreous-treated CAMs in the absence or in the presence of Boc-FLFLF; nuclei were stained with DAPI (blue). Data are means \pm SEM. $* * p<0.01$ vs untreated. Scale bars, $50 \mu \mathrm{m}$ (white) and $2 \mathrm{~mm}$ (black)

16 out of the 20 individual samples tested. Notably, intravitreal pretreatment of PDR patients with the VEGF blocker ranibizumab $10-15$ days before pars plana vitrectomy did not affect the angiogenic activity of PDR vitreous nor the inhibitory activity of UPARANT (ESM Fig. 3).

Finally, UPARANT was assessed for the capacity to suppress the inflammatory response triggered by PDR vitreous in the CAM assay. The experiments were performed on one pooled vitreous fluid sample (Fig. 6c) and on five individual PDR samples (Fig. 6d). In both cases, UPARANT inhibited the recruitment of the $\mathrm{CD} 45^{+}$infiltrate in the CAM mesenchyme surrounding the PDR vitreous-loaded plug. Again, this was paralleled by a significant inhibition of neovessel formation that was linearly related to the reduction of the inflammatory infiltrate (Fig. 6e).

\section{Discussion}

Here, the vitreous humour obtained from PDR patients after pars plana vitrectomy was used as a tool to investigate the relationship between neovascular and inflammatory responses that occur in endothelial cells during PDR.
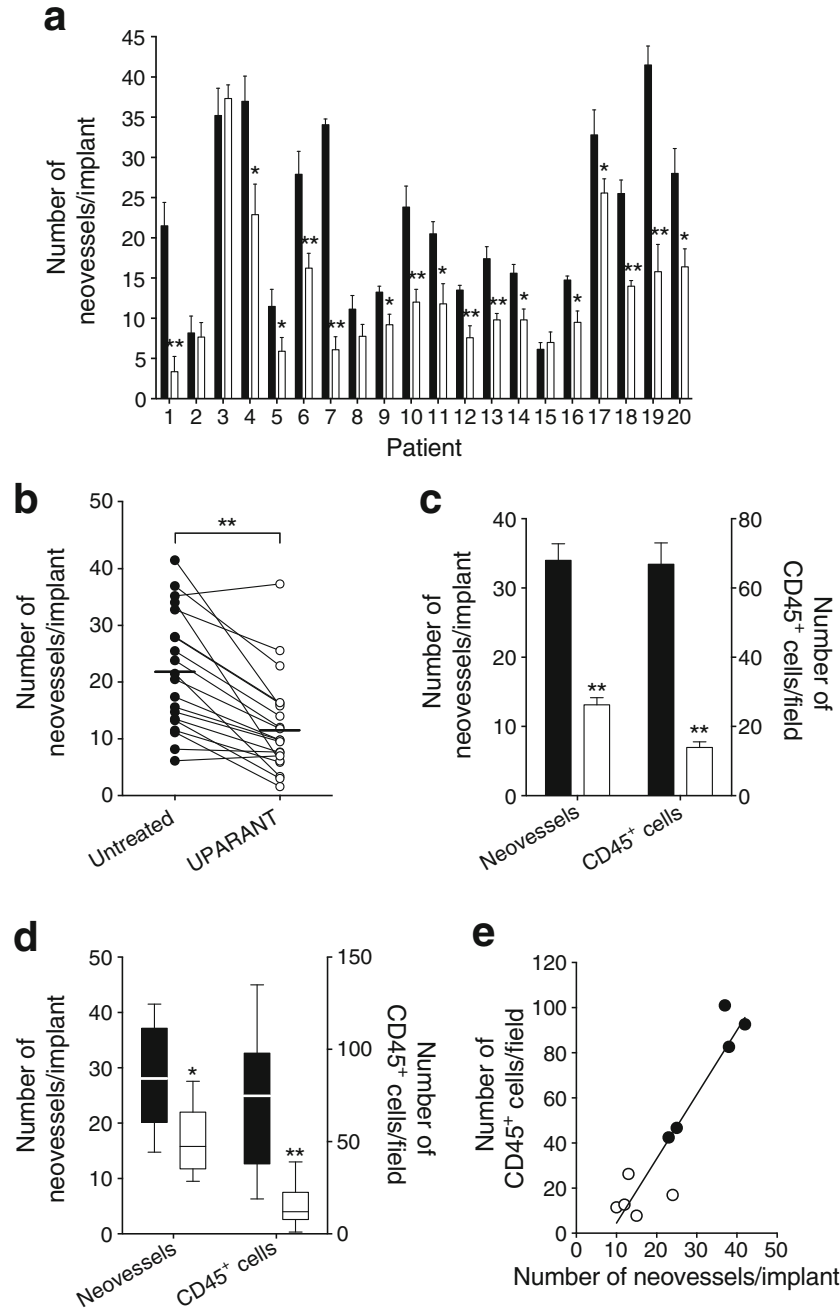

Fig. 6 UPARANT inhibits PDR vitreous-induced neovascular and inflammatory responses. $(\mathbf{a}, \mathbf{b})$ Number of neovessels in CAMs treated with 20 individual PDR vitreous samples in the absence or presence of UPARANT ( $n=8$ implants/patient). (c, d) Number of neovessels/implant $(n=8)$ and of infiltrating $\mathrm{CD} 5^{+}$cells/microscopic field $(n=25)$ in CAMs treated with one pooled PDR vitreous sample (c) or five individual PDR vitreous samples (d) in the absence or presence of UPARANT. (e) Positive correlation between the number of neovessels and of infiltrating CD $45^{+}$cells in CAMs treated with individual PDR vitreous samples in the absence or presence of UPARANT. Black symbols, PDR vitreous; white symbols, PDR vitreous + UPARANT. Data are means \pm SEM. $* p<0.05 ; * *<<0.01$ vs untreated

A variety of cytokines, chemokines and angiogenic growth factors are detectable in PDR vitreous (ESM Table 2 and Wang et al and Dai et al $[19,20])$. Accordingly, PDR vitreous activates in vitro all the steps of the angiogenic process, including endothelial cell proliferation, motility, sprouting and morphogenesis. PDR vitreous induces also a proinflammatory activation of endothelial cells characterised by the nuclear translocation of the proinflammatory transcription factors NF-kB and pCREB [27-29], ROS production, disruption of endothelial cell barrier integrity, upregulation of E-SELECTIN, VCAM-1 and ICAM-1 expression and consequent increase in leucocyte adhesion. 
In keeping with its proinflammatory/pro-angiogenic potential, PDR vitreous triggers a potent neovascular response and the recruitment of a $\mathrm{CD} 45^{+} / \mathrm{CD} 11^{+}$infiltrate in the in vivo murine Matrigel plug assay characterised by a predominant pro-angiogenic M2 polarisation. Accordingly, a rapid CD $45^{+}$ leucocyte recruitment occurs when PDR vitreous is tested for its angiogenic capacity in the chick embryo CAM assay. Notably, a significant positive correlation was observed between the number of neovessels and of infiltrating CD $45^{+}$ cells when PDR vitreous samples from different patients were individually tested in the CAM assay. Together, these data indicate that a strict correlation may exist between the proangiogenic and proinflammatory activity of PDR vitreous, the inflammatory environment playing a non-redundant role in neovessel formation. The capacity of the anti-inflammatory drug hydrocortisone to inhibit both inflammatory and angiogenic responses in PDR vitreous-treated CAMs supports this hypothesis. This is in keeping with previous observations indicating that inflammation plays a pivotal role in the angiogenesis process driven by various angiogenic growth factors, including VEGF [30], fibroblast growth factor 2 (FGF2) [31], placenta growth factor (PIGF) [32] and IL-1 $\beta$ [33].

At variance with hydrocortisone, the VEGF blocker bevacizumab was poorly effective in inhibiting the activity of PDR vitreous. This accords with previous observations showing that the K5-NOS(H) polysaccharide, a paninhibitor for heparin-binding proinflammatory/angiogenic factors, was more effective than bevacizumab in inhibiting the angiogenic activity of PDR vitreous [7]. Thus, the best performance of hydrocortisone vs bevacizumab suggests that the angio-inflammatory responses elicited by PDR vitreous may represent the result of the synergistic action of various modulators, besides VEGF.

Notably, no difference in pro-angiogenic/proinflammatory activity was observed between haemorrhagic and nonhaemorrhagic PDR vitreous samples when tested in the CAM assay (ESM Fig. 4), indicating that local production, rather than systemic inflow, is the relevant source of cytokines within the ocular PDR microenvironment. This is in keeping with the high vitreous/plasma ratio measured for cytokine levels in PDR patients [34-36]. Together, these data point to local eye inflammation as a driving force that sustains angiogenesis in PDR.

Intravitreal administration of corticosteroids is associated with possible adverse events [10,11], leading to the requirement for novel anti-inflammatory approaches in PDR therapy able to suppress retinal neovascularisation. FPRs are G protein-coupled receptors implicated in the regulation of innate immune responses, inflammation, tissue repair and angiogenesis [12]. FPRs exert a productive interaction with the $\mathrm{uPAR}_{88-92}$ receptor region that modulates the biological response of leucocytes and endothelial cells to inflammatory mediators and angiogenic factors [37-39]. On this basis, allosteric inhibitors related to the $\mathrm{uPAR}_{88-92}$ sequence and able to block the cross talk involving uPAR, FPRs and integrins were developed [16, 40, 41]. Among them, UPARANT competes with AMLF peptide for the binding to FPRs and is endowed with a significant anti-angiogenic activity in vitro and in vivo [16-18]. In addition, UPARANT suppresses the angiogenic activity of pooled PDR vitreous samples, pointing to this compound as a promising therapeutic for the treatment of inflammatory diseases associated with ocular angiogenesis, including PDR [17, 18].

Here, we demonstrate that UPARANT inhibits the neovascular response elicited in the CAM assay by 16 out of the 20 individual vitreous samples obtained from PDR patients. Inhibition of neovessel formation by UPARANT went along with a significant reduction in the inflammatory infiltrate. Notably, intravitreal pretreatment of PDR patients with the VEGF blocker ranibizumab before vitrectomy did not affect the inhibitory effect exerted by UPARANT. Together, these data strongly support the hypothesis that the inflammatory response elicited by the PDR vitreous via FPR activation plays a non-redundant role in neovessel formation and raise the question about the endothelial FPR subtype(s) and vitreous fluid mediator(s) responsible for such activation.

Our results demonstrate that HUVECs express FPR3, but not FPR1 or FPR2. Accordingly, the pan-FPR antagonist BocFLFLF hampers the angio-inflammatory responses elicited by PDR vitreous in endothelial cells, whereas the FPR1 antagonist ciclosporin $\mathrm{H}$ and the FPR2 antagonist WRW4 [12] were ineffective. However, VEGF-inducible expression of FPR2 has been reported in endothelial cells [42], indicating that differences in cell isolation and/or cell culture conditions, as well as changes of the in vivo microenvironment, may affect the pattern of FPR expression in endothelial cells. This calls for further experiments aimed to assess the expression of FPRs in retinal vessels of PDR patients.

Various danger-associated molecular pattern host-derived peptides can activate FPRs [12]. Notably, the FPR ligands serum amyloid A, LL-37 and Hp(2-20) have been involved in the regulation of neovascularisation under inflammatory conditions [12]. High levels of serum amyloid A are detectable in the vitreous and plasma of PDR patients [43] and in eyes with macular oedema $[44,45]$ whereas, to the best of our knowledge, no data are available about the levels of other FPR ligands in PDR vitreous.

Even though further studies will be required to identify unambiguously the FPR subtypes and their natural ligands acting as mediators of the angiogenic/inflammatory activity of PDR vitreous, our data indicate that anti-angiogenic strategies targeting FPR activation may be exploited in persistent ocular inflammatory conditions, including PDR. In this frame, UPARANT may represent the basis for the development of novel anti-inflammatory/anti-angiogenic approaches for PDR therapy. 
Acknowledgements We thank S. Calza (Department of Molecular and Translational Medicine, University of Brescia, Italy) for statistical analysis and S. Liekens (Rega Institute, Leuven, Belgium) for trans-endothelial electrical resistance measurements.

Data availability The authors declare that the data supporting the findings of this study are available within the article and its supplementary information file.

Funding This work was supported in part by grants from: Ministero dell'Istruzione, Università e Ricerca (FIRB project RBAP11H2R9 2011), Associazione Italiana per la Ricerca sul Cancro (AIRC grant no. 14395) and BIOOS Italia to MP; Fondi Europei per lo Sviluppo Regionale, Ministero dell'Istruzione, Università e Ricerca and Ministero dello Sviluppo Economico (PON01 02464) to MDR and VP; AIRC IG 2015, grant 17276 to SM, SR and PC were supported by fellowships from AIRC and Fondazione Italiana per la Ricerca sul Cancro (FIRC), respectively.

Duality of interest The authors declare that there is no duality of interest associated with this manuscript.

Contribution statement SR contributed to study design, acquisition and analysis of data and drafted the manuscript. MC, PC, AC, IMN, DC, SM, RR, MB and LL contributed to study design, acquisition and analysis of data and critical revision of the manuscript. LL performed the synthesis of UPARANT and contributed to acquisition and analysis of data. DR, MDR, VP and FS contributed to the conception and design of the experiments, interpretation of data and critical revision of the manuscript. MP supervised the project, designed experiments, analysed data and drafted the manuscript. All authors gave their approval to the final version of the manuscript. MP is responsible for the integrity of the work as a whole.

\section{References}

1. Congdon N, O'Colmain B, Klaver CC et al (2004) Causes and prevalence of visual impairment among adults in the United States. Arch Ophthalmol 122:477-485

2. Bandello F, Lattanzio R, Zucchiatti I, Del Turco C (2013) Pathophysiology and treatment of diabetic retinopathy. Acta Diabetol 50:1-20

3. Wilkinson CP, Ferris FL 3rd, Klein RE et al (2003) Proposed international clinical diabetic retinopathy and diabetic macular edema disease severity scales. Ophthalmology 110:1677-1682

4. Stitt AW, Curtis TM, Chen M et al (2016) The progress in understanding and treatment of diabetic retinopathy. Prog Retin Eye Res $51: 156-186$

5. Tang J, Kern TS (2011) Inflammation in diabetic retinopathy. Prog Retin Eye Res 30:343-358

6. Kwong TQ, Mohamed M (2014) Anti-vascular endothelial growth factor therapies in ophthalmology: current use, controversies and the future. Br J Clin Pharmacol 78:699-706

7. Rezzola S, Dal Monte M, Belleri M et al (2015) Therapeutic potential of anti-angiogenic multitarget N, O-sulfated E. coli K5 polysaccharide in diabetic retinopathy. Diabetes 64:2581-2592
8. Tas SW, Maracle CX, Balogh E, Szekanecz Z (2016) Targeting of proangiogenic signalling pathways in chronic inflammation. Nat Rev Rheumatol 12:111-122

9. Semeraro F, Cancarini A, dell'Omo R, Rezzola S, Romano MR, Costagliola C (2015) Diabetic retinopathy: vascular and inflammatory disease. J Diabetes Res 2015:582060

10. Ahmadieh H, Feghhi M, Tabatabaei H, Shoeibi N, Ramezani A, Mohebbi MR (2008) Triamcinolone acetonide in silicone-filled eyes as adjunctive treatment for proliferative vitreoretinopathy: a randomized clinical trial. Ophthalmology 115:1938-1943

11. Diabetic Retinopathy Clinical Research Network (2008) A randomized trial comparing intravitreal triamcinolone acetonide and focal/ grid photocoagulation for diabetic macular edema. Ophthalmology 115:1447-1449

12. Prevete N, Liotti F, Marone G, Melillo RM, de Paulis A (2015) Formyl peptide receptors at the interface of inflammation, angiogenesis and tumor growth. Pharmacol Res 102:184-191

13. Waltz DA, Fujita RM, Yang X et al (2000) Nonproteolytic role for the urokinase receptor in cellular migration in vivo. Am J Respir Cell Mol Biol 22:316-322

14. Duru EA, Fu Y, Davies MG (2015) Role of formic receptors in soluble urokinase receptor-induced human vascular smooth muscle migration. J Surg Res 195:396-405

15. Gargiulo L, Longanesi-Cattani I, Bifulco K et al (2005) Cross-talk between fMLP and vitronectin receptors triggered by urokinase receptor-derived SRSRY peptide. J Biol Chem 280:25225-25232

16. Carriero MV, Bifulco K, Minopoli M et al (2014) UPARANT: a urokinase receptor-derived peptide inhibitor of VEGF-driven angiogenesis with enhanced stability and in vitro and in vivo potency. Mol Cancer Ther 13:1092-1104

17. Dal Monte M, Rezzola S, Cammalleri M et al (2015) Antiangiogenic effectiveness of the urokinase receptor-derived peptide UPARANT in a model of oxygen-induced retinopathy. Invest Ophthalmol Vis Sci 56:2392-2407

18. Cammalleri M, Dal Monte M, Locri F et al (2016) The urokinase receptor-derived peptide UPARANT mitigates angiogenesis in a mouse model of laser-induced choroidal neovascularization. Invest Ophthalmol Vis Sci 57:2586-2597

19. Wang S, Park JK, Duh EJ (2012) Novel targets against retinal angiogenesis in diabetic retinopathy. Curr Diab Rep 12:355-363

20. Dai Y, Wu Z, Wang F, Zhang Z, Yu M (2014) Identification of chemokines and growth factors in proliferative diabetic retinopathy vitreous. Biomed Res Int 2014:486386

21. Aiello LP, Avery RL, Arrigg PG et al (1994) Vascular endothelial growth factor in ocular fluid of patients with diabetic retinopathy and other retinal disorders. N Engl J Med 331:1480-1487

22. Takagi H, Watanabe D, Suzuma K et al (2007) Novel role of erythropoietin in proliferative diabetic retinopathy. Diabetes Res Clin Pract 77(Suppl 1):S62-S64

23. Murugeswari P, Shukla D, Kim R, Namperumalsamy P, Stitt AW, Muthukkaruppan V (2014) Angiogenic potential of vitreous from proliferative diabetic retinopathy and Eales' disease patients. PLoS One 9, e107551

24. Coltrini D, Di Salle E, Ronca R, Belleri M, Testini C, Presta M (2013) Matrigel plug assay: evaluation of the angiogenic response by reverse transcription-quantitative PCR. Angiogenesis 16:469 477

25. Jablonski KA, Amici SA, Webb LM et al (2015) Novel markers to delineate murine M1 and M2 macrophages. PLoS One 10, $\mathrm{e} 0145342$

26. Deryugina EI, Quigley JP (2008) Chick embryo chorioallantoic membrane model systems to study and visualize human tumor cell metastasis. Histochem Cell Biol 130:1119-1130

27. Corsini M, Moroni E, Ravelli C et al (2014) Cyclic adenosine monophosphate-response element-binding protein mediates the 
proangiogenic or proinflammatory activity of gremlin. Arterioscler Thromb Vasc Biol 34:136-145

28. Jeon SH, Chae BC, Kim HA et al (2007) The PKA/CREB pathway is closely involved in VEGF expression in mouse macrophages. Mol Cells 23:23-29

29. Sehnert B, Burkhardt H, Wessels JT et al (2013) NF-kB inhibitor targeted to activated endothelium demonstrates a critical role of endothelial NF-kB in immune-mediated diseases. Proc Natl Acad Sci U S A 110:16556-16561

30. Cursiefen C, Chen L, Borges LP et al (2004) VEGF-A stimulates lymphangiogenesis and hemangiogenesis in inflammatory neovascularization via macrophage recruitment. J Clin Invest 113:1040 1050

31. Andres G, Leali D, Mitola S et al (2009) A pro-inflammatory signature mediates FGF2-induced angiogenesis. J Cell Mol Med 13: 2083-2108

32. Pipp F, Heil M, Issbrucker K et al (2003) VEGFR-1-selective VEGF homologue PIGF is arteriogenic: evidence for a monocytemediated mechanism. Circ Res 92:378-385

33. Nakao S, Kuwano T, Tsutsumi-Miyahara $C$ et al (2005) Infiltration of COX-2-expressing macrophages is a prerequisite for IL-1 betainduced neovascularization and tumor growth. J Clin Invest 115: 2979-2991

34. Gustavsson C, Agardh CD, Agardh E (2013) Profile of intraocular tumour necrosis factor-alpha and interleukin-6 in diabetic subjects with different degrees of diabetic retinopathy. Acta Ophthalmol 91: 445-452

35. Semeraro F, Cancarini A, Morescalchi F et al (2014) Serum and intraocular concentrations of erythropoietin and vascular endothelial growth factor in patients with type 2 diabetes and proliferative retinopathy. Diabetes Metab 40:445-451
36. Takeuchi M, Sato T, Tanaka A et al (2015) Elevated levels of cytokines associated with Th2 and Th17 cells in vitreous fluid of proliferative diabetic retinopathy patients. PLoS One 10, e0137358

37. Selleri C, Montuori N, Ricci P et al (2005) Involvement of the urokinase-type plasminogen activator receptor in hematopoietic stem cell mobilization. Blood 105:2198-2205

38. Resnati M, Pallavicini I, Wang JM et al (2002) The fibrinolytic receptor for urokinase activates the $\mathrm{G}$ protein-coupled chemotactic receptor FPRL1/LXA4R. Proc Natl Acad Sci U S A 99:1359-1364

39. Bifulco K, Longanesi-Cattani I, Liguori E et al (2013) A urokinase receptor-derived peptide inhibiting VEGF-dependent directional migration and vascular sprouting. Mol Cancer Ther 12:1981-1993

40. Bifulco K, Longanesi-Cattani I, Gargiulo L et al (2008) An urokinase receptor antagonist that inhibits cell migration by blocking the formyl peptide receptor. FEBS Lett 582:1141-1146

41. Carriero MV, Longanesi-Cattani I, Bifulco K et al (2009) Structurebased design of an urokinase-type plasminogen activator receptorderived peptide inhibiting cell migration and lung metastasis. Mol Cancer Ther 8:2708-2717

42. Lee MS, Ghim J, Kim SJ et al (2015) Functional interaction between CTGF and FPRL1 regulates VEGF-A-induced angiogenesis. Cell Signal 27:1439-1448

43. Ma Y, Tao Y, Lu Q, Jiang YR (2011) Intraocular expression of serum amyloid a and interleukin-6 in proliferative diabetic retinopathy. Am J Ophthalmol 152(678-685), e672

44. Feng J, Zhao T, Zhang Y, Ma Y, Jiang Y (2013) Differences in aqueous concentrations of cytokines in macular edema secondary to branch and central retinal vein occlusion. PLoS One 8, e68149

45. Wen J, Jiang Y, Zheng X, Zhou Y (2015) Six-month changes in cytokine levels after intravitreal bevacizumab injection for diabetic macular oedema and macular oedema due to central retinal vein occlusion. Br J Ophthalmol 99:1334-1340 